\title{
FERRAMENTA EDUCACIONAL TDAHMENTE - APLICATIVO INDICADOR DE DIAGNÓSTICO TDAH EM CRIANÇAS E ADOLESCENTES
}

\author{
PORTO VELHO/RO JUNHO/2018
Cleiton Aparecido de Araújo Afonso - ICD - cleiton1985@gmail.com
Neide Borges Pedrosa - UNIR - neibpedrosa@gmail.com
Rogéria Moreira Rezende Isobe - UFTM - rogeriaisobe@gmail.com \\ Tipo: Relato de Experiência Inovadora (EI) \\ Categoria: Métodos e Tecnologias \\ Setor Educacional: EDUCAÇÃO MÉDIA E TECNOLÓGICA
}

\begin{abstract}
RESUMO
Este trabalho apresenta o relato de experiência de construção do TDAHMENTE, aplicativo que se configura como ferramenta educacional para profissionais da educação com o objetivo de diagnosticar o Transtorno de Déficit de Atenção e Hiperatividade (TDAH) em crianças e adolescentes. O aplicativo foi desenvolvido a partir do ensino de linguagem de programação a alunos do Ensino Médio, criação de artes gráficas e pesquisas bibliográficas ao referencial teórico cientifico. Utilizando ferramentas e 0 espaço do PROINFO na escola, foram realizados estudos para subsidiar a construção do aplicativo tendo como referência o Manual Diagnóstico e Estatístico de Transtornos Mentais da Associação Americana de Psiquiatria (DSM), que habilita profissionais médicos a diagnosticar o TDAH através de formulários específicos. Com base nesse material foi construído um aplicativo digital que não substitui o laudo médico, mas que se configura como um indicador de diagnóstico oferecendo ao usuário estratégias de autoconhecimento bem como a possibilidade de procurar um profissional médico a partir dos resultados obtidos com a ferramenta. Acredita-se que a criação e publicação dessa ferramenta inovadora possa contribuir para orientação de profissionais da educação e pais de alunos no diagnóstico do TDAH, que se inscreve no conjunto das problemáticas educacionais que podem conduzir ao fracasso escolar na contemporaneidade.
\end{abstract}

Palavras-chave: Educação, ferramenta educacional, TDAH, aplicativo digital 


\section{Introdução}

O projeto de construção do aplicativo TDAHMENTE resulta de um trabalho desenvolvido por alunos do Ensino Médio do Instituto Estadual de Educação Carmela Dutra - Porto Velho-RO, sob a coordenação do professor de informática da Instituição com a participação e orientação de docentes da Universidade Federal de Rondônia - UNIR e Universidade Federal do Triângulo Mineiro - UFTM. Utilizando ferramentas e espaço do Programa Nacional de Tecnologia Educacional (PROINFO), foi criado o aplicativo digital TDAHMENTE, uma ferramenta digital com o objetivo de identificar possível diagnóstico do Transtorno de Déficit de Atenção e Hiperatividade (TDAH) em alunos da educação básica, auxiliando pais de alunos, professores, coordenadores e psicólogos. O aplicativo foi desenvolvido a partir do ensino de linguagem de programação a alunos do Ensino Médio, criação de artes gráficas e pesquisas bibliográficas ao referencial teórico cientifico.

O aplicativo TDAHMENTE possibilita aos usuários apropriação de autoconhecimento sobre o TDAH que consiste em uma desordem comportamental com base neurológica frequente na infância (RICHTERS, et., all, 1995) caracterizado por graus diferenciados de desatenção, impulsividade e, em alguns casos, hiperatividade (CHADD, 2001 apud FERREIRA, 2011) . De acordo com Lacet e Rosa (2017) a pesquisa com 5.961 jovens de 18 estados do país, identificou que no cenário nacional $4,4 \%$ da população de crianças e adolescentes entre 04 e 18 anos de idade possuem TDAH, trata-se do primeiro estudo epidemiológico sobre TDAH feito no Brasil com abrangência em todo o território. O índice se assemelha aos observados em outras partes do mundo, que indicam a presença de TDAH em $5 \%$ dos jovens. A maior problemática em tratar 0 TDAH é a falta de diagnóstico, e o TDAHMENTE surgiu, nessa conjuntura, como proposta de ferramenta alternativa para auxiliar as pessoas a ter conhecimento e buscar identificar um possível diagnóstico para os transtornos de atenção e hiperatividade.

As pesquisas que nortearam a criação do aplicativo revelaram que os sintomas do TDAH permanecem na vida adulta em $67 \%$ dos casos (LOPES, NASCIMENTO e BANDEIRA, 2005) e trazem implicações à rotina da criança e da família, bem como consequências ao sistema educacional e maior incidência de condutas de risco na adolescência (HERNÁNDEZ, 2007). Frequentemente relaciona-se ao insucesso educacional, baixa performance profissional, perda na renda familiar, impacto econômico e social (BIEDERMAN e FARAONE, 2006; ROHDE e HALPERN, 2004).

Para fundamentação do aplicativo foi utilizado como referência os dados disponíveis no Manual Diagnóstico e Estatístico de Transtorno Mentais (DSM, 2014), um conjunto de 
questões formuladas pela Associação Americana de Psiquiatria que permite apenas ao profissional médico diagnosticar o TDAH. A transformação do DSM em um formulário digital como aplicativo não substitui o profissional médico, mas o torna uma ferramenta auxiliar que busca fornecer informações baseadas em pesquisas cientificas que resultem indicadores potenciais para um nível elevado do transtorno. O Indicador de TDAH é uma ferramenta e recurso indispensável para que o usuário possa se apropriar de conhecimento e avaliar a possível necessidade de procurar ajuda de um profissional médico de acordo com os resultados obtidos no aplicativo. O projeto é inovador pois substitui os velhos métodos tradicionais e defasados que ainda são utilizados por profissionais da educação na busca de identificar possível diagnóstico TDAH em crianças e adolescentes no âmbito escolar, pois, muitos formulários utilizados atualmente são reproduções da internet e possuem metodologia questionável. $O$ TDAHMENTE possui respaldo científico na medida em que foi construído com base no DSM. Além das pesquisas científicas realizadas pelos pesquisadores o desenvolvimento do aplicativo contou com a colaboração de um profissional médico de psiquiatria que nos ofereceu todos os recursos e suporte para o desenvolvimento da ferramenta.

O trabalho é inovador como projeto científico e impactante como ferramenta educacional, visto que não há nada semelhante nos sítios de internet. O aplicativo foi construído seguindo várias etapas metodológicas, desde o referencial teórico científico que fundamenta a aplicação e os procedimentos metodológicos que utilizaram técnicas de conceituados autores como Meurer (2014), Santos (2005) e Lobach (2007).

\section{Referencial Teórico}

Com as novas tecnologias da informação conectadas à internet, a escola precisa cada vez superar os desafios e romper as fronteiras digitais se inserindo na realidade social da comunidade escolar, do cotidiano do aluno, compartilhando, socializando experiências, oportunizando que aprendizagem seja além da sala de aula, uma prática contínua e acessível a comunidade em geral. Nessa conjuntura "a escola sai do seu casulo, do seu mundinho e se torna uma instituição onde a comunidade pode aprender contínua e flexivelmente (MORAN, 2007, p.12)

Com as diversas ferramentas gratuitas e disponíveis na rede mundial de computadores é possível desenvolver, criar e divulgar novas aplicações, assim como utilizar os laboratórios de informática escolares não apenas como espaço de pesquisas tradicionais, mas aproveitar as ferramentas disponíveis para o desenvolvimento de novas tecnologias. 
Partindo da vivência com alunos de TDAH no ambiente escolar, bem como da dificuldade em diagnosticar corretamente pessoas portadoras do transtorno de déficit de atenção, hiperatividade e impulsividade, notou-se a necessidade de buscar referências teóricas que fundamentassem toda a pesquisa.

Os procedimentos de diagnóstico de TDAH seguem um referencial cientifico estabelecido e seguido por profissionais da área médica do mundo todo - o DSM - que consiste no norteador de toda a pesquisa. Visa trazer respaldo científico e desmistificar formulários e referências questionáveis que são encontrados na rede mundial de computadores. O DSM é fundamental e essencial para a fundamentação científica do aplicativo TDAHMENTE, sugerindo estudos, pesquisa, análise e interpretação do orientador e aluno pesquisador. Larroca e Domingos (2012) afirmam que o

O diagnóstico do TDAH é determinado mediante a satisfação dos critérios estabelecidos pelo DSM-IV, (...), esses sintomas são observados considerando-se a persistência de sua manifestação e sua severidade em relação aos comportamentos tipicamente observados em indivíduos de nível equivalente de desenvolvimento (p. 114, grifos no original).

Embora haja controvérsias quanto ao diagnóstico TDAH na comunidade científica, o DSM é o único referencial teórico científico com critérios estabelecidos pela Associação Americana de Psiquiatria confiável para elaboração de formulários que são utilizados pelo médico, mas utilizados como referência por outros setores da sociedade.

Partindo do pressuposto que só o médico pode diagnosticar o TDAH, o aplicativo TDAHMENTE, apresenta-se como um indicador embasado em relatórios e pesquisas médicas com finalidade de sugerir um questionário capaz de indicar um potencial diagnóstico de TDAH em crianças e adolescentes. Configura-se, portanto, como um meio digital de fornecer informações baseadas em critérios científicos, possibilitando uma análise do comportamento individual que possa indicar a necessidade de buscar ajuda médica. O aplicativo torna-se, pois, fundamental para profissionais da educação, pois pode ajudar na análise comportamental de alunos que apresentam dificuldades em manter e fixar a atenção, dificuldades com a leitura e a escrita, entre outras.

A prática de pesquisa e inovação para os alunos do Ensino Médio é algo muito recente sendo que no Estado de Rondônia a iniciação cientifica se apresenta como proposta curricular explicita no Referencial Curricular do Estado e como política de incentivo, através da Gerência do Ensino Médio promovida pela Secretaria de Educação (SEDUC), fomentando assim a motivação em professores e alunos a praticarem 
pesquisa e divulgar seus trabalhos através da Feira de Rondônia de Inovação e Tecnologia. O projeto TDAHMENTE é resultado das políticas promovidas pela Secretaria de Estado de Educação do Estado de Rondônia a partir do ensino de programação no laboratório de informática da escola.

\section{Procedimentos Metodológicos}

Os procedimentos adotados fundamentaram-se no Projeto E (MEURER, 2014) voltado para o desenvolvimento de produtos digitais, baseando-se na compilação de vários processos, técnicas e métodos para a produção de aplicações. Utilizou-se os planos definidos no projeto com referências a Garret (2003) da área de tecnologia da informação e comunicação, bem como Lobach (2007) e Gomes (2011) que muito agregaram à execução do projeto, devido a complexidade do tema abordado.

As etapas metodológicas de acordo com (Meurer 2014) seguiram os seguintes critérios: contextualização: que teve como objetivo espacializar o TDAH no cenário nacional e mundial como transtorno mental; identidade: buscando criar aspectos gráficos e interface de fácil compreensão e relação com os transtornos que ocorrem no cérebro humano, dando origem a criação do logotipo e interface; comparação quando buscouse o estudo de outras aplicações com finalidade de diferenciar o produto projetado como inovação tecnológica; desconstrução que teve como objetivo coletar dados de similares para análises e comparações; verificação por meio da análise da opinião pública de usuários acerca de similares já desenvolvidos; reconstrução a partir de todas as análises realizadas buscou-se construir a ferramenta tecnologia com respaldo teórico cientifico para suprir as demandas de usuários que buscam identificar um possível diagnóstico TDAH.

Para a estruturação e organização das etapas de desenvolvimento utilizou-se o Método de Desdobramento em 3 etapas criado por Santos (2005), o MD3E, que fundamentou as seguintes etapas: Pré-Concepção: compreendeu a todas as fases de estudos bibliográficos que permitiram idealizar a projeção final; Concepção consistiu em todas as ferramentas e técnicas práticas utilizadas para a produção; Pós-concepção consiste em todas as atividades em curso que estão sendo desenvolvidas após o lançamento da ferramental digital como protótipo, é o momento de análises de resultados, elaboração de relatórios, divulgação, participação em feiras científicas e congressos.

O projeto foi desenvolvido no laboratório de informática da instituição PROINFO Programa Nacional de Tecnologia Educacional, disponível no Instituto Estadual de Educação Carmela Dutra. A metodologia seguiu seis etapas a partir do referencial de 
Meurer (2014) e Santos (2005), sendo a primeira etapa a revisão bibliográfica do marco teórico cientifico com a elaboração do questionário com fundamentação cientifica, bem como a análise de artigos, periódicos e livros publicados referentes ao TDAH. Na segunda etapa foi ministrado aulas práticas de linguagem de programação e instaladas a plataforma básica do sistema. Na terceira etapa o desenvolvimento e incorporação de códigos fonte livres como arquivos do projeto. Na quarta, realizado testes e comparações com códigos livres já instalados na plataforma. Na quinta etapa, realizado a otimização para melhor desempenho, aplicações para visualização em aparelhos móbiles e aplicação para a plataforma Android. Na sexta etapa, produção do relatório final e divulgação.

Com a adoção do DSM como questionário para indicar possível diagnóstico de TDAH, utilizou-se na ferramenta de programação a avaliação heurística (NIELSEN, 1995). Esta avaliação é um processo que busca compreender a interação entre o homem e o computador a partir de uma interface digital. Utilizou-se a análise sincrônica e diacrônica dos resultados (BIONSIEPE et., all., 1984), expondo uma morfologia, estrutural, funcional e gráfico visual, conforme postulados de Nielsen (1995).

Foi utilizado para como referência morfológica os estudos de Pazmino (2010), a partir da matriz ou caixa morfológica que possibilitou a sistematização visual dos conceitos definindo, assim, as plataformas, sistemas operacionais, a tipografia, a ilustração. A partir de todos os processos aplicados, conforme Santos (2005), foi finalizado a criação da ferramenta final.

A ferramenta digital foi submetida a análise de um profissional competente, o médico psiquiatra Dr. Robinson C. Machado CRM 1188-RO / RQE 1696, o qual está colaborando com a fase experimental de testes do aplicativo e irá expedir um relatório médico sobre as funcionalidades do aplicativo digital após os testes com pacientes que utilizaram o aplicativo, atestando a ferramenta como indicador de possível diagnóstico de TDAH para profissionais da Educação, reiterando que o mesmo não substitui o diagnóstico médico. O aplicativo TDAHMENTE está sendo distribuído como ferramenta de Indicador de Diagnóstico TDAH na PlayStore do Google para testes e aperfeiçoamento das suas funcionalidades, tendo a aceitação e participação de público geral.

\section{Resultados}

Com a disponibilidade da ferramenta no sítio de aplicativos da PlayStore do Google, o aplicativo tem ganhado notoriedade da sociedade, sendo avaliado positivamente por 
centenas de usuários que já baixaram o aplicativo em seus aparelhos smartphones, além de disponível como aplicativo android. O TDAHMENTE está disponível para desktop e responsivo para outras plataformas como IOS e Windows Phone e tem sido bastante visualizado devido divulgação em canais de notícias. No ambiente escolar o aplicativo está sendo testado por professores e alunos que estão tendo conhecimento do TDAH. A fase de testes tem sido essencial para ajustamento de funcionalidades e futuras implementações de recursos que são sugeridos por usuários.

A ferramenta tem sido propulsora para difundir o conhecimento do TDAH sendo que as participações em diversos meios de comunicação permitiram a adesão de parceiros, psiquiatras, psicólogos, profissionais da educação e outras instituições que querem participar do desenvolvimento da ferramenta tecnológica. Além de ser uma ferramenta de utilidade pública, a execução do projeto mostra para a sociedade que a pesquisa científica se faz presente na educação básica, despertando a motivação e interesse pelo conhecimento cientifico em outros profissionais da educação básica.

\section{Considerações Finais}

A educação tecnológica com ensino de programação na educação básica possibilitou através da vivência escolar com alunos com TDAH o surgimento de uma tecnologia inovadora na rede mundial de computadores. O TDAHMENTE, é uma inovação, pois oportuniza através de metodologia cientifica oportunizar uma ferramenta gratuita de auto apropriação de conhecimento do transtorno de déficit de atenção e hiperatividade, possibilitando o indivíduo portador de TDAH o seu reconhecimento e busca de um diagnóstico através do profissional médico.

Consideramos um trabalho de relevância social na medida em que grande parte de crianças e adolescentes com TDAH não são diagnosticadas por falta de conhecimento, trazendo consequências ao sistema educacional, problemas de condutas comportamentais na adolescência, vítimas de bullying, violência, depressão e baixo rendimento escolar. O projeto é impactante, pois além de contribuir com o diagnóstico oportuniza esclarecimento a partir de uma plataforma atraente e lúdica.

O TDAHMENTE atraiu os holofotes da mídia local trazendo para dentro do projeto profissionais interessados em contribuir com a temática, tais como psicólogos, psiquiatras, pedagogos, especialistas em educação e pesquisadores possibilitando ampliar os recursos e ferramentas, tornando uma plataforma de desenvolvimento. Embora o aplicativo não substitua o diagnóstico médico ele está se tornando uma referência nos sítios em que está disponível devido sua metodologia desenvolvida. 
Buscaremos a continuidade das pesquisas e o aperfeiçoamento com a implantação de novos recursos no aplicativo.

\section{Bibliografia}

BIEDERMAN, J., e FARAONE, S. V. (2006). The effects of AttentionDeficit/Hyperactivity Disorder on Employment and Household Income. Medscape General Medicine, 8(3), 12.

BONSIEPE, G., KELLNER, P. e POESSNECKER, H. Método experimental: Desenho Industrial. Florianópolis: LDP/DI, 1984.

DSM. Manual diagnóstico e estatístico de transtornos mentais [recurso eletrônico] : DSM-5 / [American Psychiatric Association ; tradução: Maria Inês Corrêa Nascimento ... et al.] ; revisão técnica: Aristides Volpato Cordioli ... [et al.]. - 5. ed. - Dados eletrônicos. - Porto Alegre: Artmed, 2014. Disponível em: https://aempreendedora.com.br/wp-conten t/uploads/2017/04/Manual-Diagn\%C3\%B3stico-e-Estat\%C3\%ADstico-de-TranstornosMentais-DSM-5.pdf Acessado em 10/10/2017

FERREIRA, P. V. C. Uma Revisão Teórica sobre o Transtorno de Déficit de Atenção e Hiperatividade (TDAH) e Estratégias Educacionais de Atendimento ao Aluno com TDAH. Revista de Psicologia, Fortaleza, v. 2 n. 2, p. 57-75, jul./dez. 2011

GARRET, J. J. The Elements os Use Experience: User Center Design for The Web. New Riders, 2003.

GOMES, L. A. V. N. Criatividade e design: um livro de desenho industrial para projeto de produto. Porto Alegre: sCHDs, 2011.

LOBACH, B. Design industrial: bases para a configuração dos produtos industriais. s. I.: Edgar Blücher, 2007.

HERNÁNDEZ, G. M. C. Transtorno por déficit de atención e hiperactividad. Revista Peruana de Pediatria, 60(2), 126-131. 2007.

LACET, C. e ROSA, M. D. Diagnóstico de Transtorno de Déficit de Atenção e Hiperatividade (TDAH) e sua história no discurso social: desdobramentos subjetivos e éticos - Revista de Psicologia-PUC. São Paulo, volume 26, n.2, 231-253, 2017. 
LARROCA, L. M. e DOMINGOS, N. M. TDAH - Investigação dos critérios para diagnóstico do subtipo predominantemente desatento. Revista Semestral da Associação Brasileira de Psicologia Escolar e Educacional, SP. Volume 16, Número 1, Janeiro/Junho de 2012: 113-123.Disponível em http://www.scielo.br/scielo.php?script=sci_arttext\&pid=S1413-85572012000100012 acessado em 10/11/2017

LOPES, R. M; NASCIMENTO, R.F.L; BANDEIRA, D.R. Avaliação do transtorno de déficit de atenção/hiperatividade em adultos (TDAH): uma revisão de literatura. Aval. Psicol., jun. 2005, vol. 4, no 1, p. 65-74. ISSN 1677-0471. Disponível em: http://pepsic.bvs-psi.org.br/scielo.php?script=sciartext\&pid=s167704712005000100008\&lng=pt\&nrm=iso. Acesso em: 09/11/2017.

MEURER, H. Ferramenta de gerenciamento e recomendação como recurso na aprendizagem baseada em projeto de design. Tese de Doutorado do Programa de Pós-Graduação em Informática na Educação da Universidade Federal do Rio Grande do Sul (UFRGS). 2014.

MORAN C., A Educação que desejamos: Novos desafios e como chegar lá. $2^{\underline{a}}$ ed. Campinas, SP: papiros Editora, 2007, v. 1. p.174.

NIELSEN, J. 10 Usability Heuristics for User Interface Design. Nielsen Norman Group, 1995

PAZMINO, A. V.. Modelo de Ensino de Métodos de Design de Produtos. Tese de Doutorado, Programa de Pós-Graduação em Design da Pontifícia Universidade Católica do Rio de Janeiro (PUC-Rio). 2010.

RICHTERS, J. E., GUIMARÃES, A. B., JENSEN, P. E., ABIKOFF, H., CONNERS, K., GREENHILL, L. L., HECHTMAN, C.. (1995). NIMH Colaborative Multisite Multimodal Treatment Study of Children with ADHD: I. Background and Rationale. Journal of the American Academy of Children and Adolescent Psychiatry, 34(8), 987-1000.

ROHDE, L. A. e HALPERN, R. Transtorno de déficit de atenção / hiperatividade: atualização. Jornal de Pediatria, 80 (2, supl): s61-s70. 2004

SANTOS, F. V. dos. MD3E (Método de Desdobramento em 3 Etapas): uma proposta de método aberto de projeto para uso no ensino de design industrial. Tese de Doutorado. Programa de Pós-Graduação em Engenharia de Produção. 2005. 
